PROCEEDINGS OF THE

AMERICAN MATHEMATICAL SOCIETY

Volume 129, Number 2, Pages 543-549

S 0002-9939(00)05566-0

Article electronically published on July 27, 2000

\title{
SIMPLE COMPLETE BOOLEAN ALGEBRAS
}

\author{
THOMAS JECH AND SAHARON SHELAH
}

(Communicated by Carl G. Jockusch, Jr.)

\begin{abstract}
For every regular cardinal $\kappa$ there exists a simple complete Boolean
algebra with $\kappa$ generators.
\end{abstract}

\section{INTRODUCTION}

A complete Boolean algebra is simple if it is atomless and has no nontrivial proper atomless complete subalgebra. The problem of the existence of simple complete Boolean algebras was first discussed in 1971 by McAloon in [8. Previously, in 7], McAloon constructed a rigid complete Boolean algebra; it is easily seen that a simple complete Boolean algebra is rigid. In fact, it has no non-trivial one-to-one complete endomorphism [1. Also, if an atomless complete algebra is not simple, then it contains a non-rigid atomless complete subalgebra [2].

McAloon proved in $[8$, that an atomless complete algebra $B$ is simple if and only if it is rigid and minimal, i.e. the generic extension by $B$ is a minimal extension of the ground model. Since Jensen's construction [5] yields a definable real of minimal degree over $L$, it shows that a simple complete Boolean algebra exists under the assumption $V=L$. McAloon then asked whether a rigid minimal algebra can be constructed without such an assumption.

In [10, Shelah proved the existence of a rigid complete Boolean algebra of cardinality $\kappa$ for each regular cardinal $\kappa$ such that $\kappa^{\aleph_{0}}=\kappa$. Neither McAloon's nor Shelah's construction gives a minimal algebra.

In [9, Sacks introduced perfect set forcing, to produce a real of minimal degree. The corresponding complete Boolean algebra is minimal, and has $\aleph_{0}$ generators. Sacks' forcing generalizes to regular uncountable cardinals $\kappa$ (cf. [6]), thus giving a minimal complete Boolean algebra with $\kappa$ generators. The algebras are not rigid however.

Under the assumption $V=L$, Jech constructed in [3] a simple complete Boolean algebra of cardinality $\kappa$, for every regular uncountable cardinal that is not weakly compact (if $\kappa$ is weakly compact, or if $\kappa$ is singular and GCH holds, then a simple complete Boolean algebra does not exist).

In [4], we proved the existence of a simple complete Boolean algebra (in ZFC). The algebra is obtained by a modification of Sacks' forcing, and has $\aleph_{0}$ generators

Received by the editors January 13, 1999 and, in revised form, April 30, 1999.

1991 Mathematics Subject Classification. Primary 03Exx.

The authors were supported in part by National Science Foundation grants DMS-98-02783 and DMS-97-04477. 
(the forcing produces a definable minimal real). The present paper gives a construction of a simple complete Boolean algebra with $\kappa$ generators, for every regular uncountable cardinal $\kappa$.

Main Theorem. Let $\kappa$ be a regular uncountable cardinal. There exists a forcing notion $P$ such that the complete Boolean algebra $B=B(P)$ is rigid, $P$ adds a subset of $\kappa$ without adding any bounded subsets, and for every $X \in V[G]$ (the P-generic extension), either $X \in V$ or $G \in V[X]$. Consequently, $B$ is a simple complete Boolean algebra with $\kappa$ generators.

The forcing $P$ is a modification of the generalization of Sacks' forcing described in [6].

\section{FORCING WITH PERFECT $\kappa$-TREES}

For the duration of the paper let $\kappa$ denote a regular uncountable cardinal, and set Seq $=\bigcup_{\alpha<\kappa}{ }^{\alpha} 2$.

Definition 2.1. (a) If $p \subseteq$ Seq and $s \in p$, say that $s$ splits in $p$ if $s \frown 0 \in p$ and $s \frown 1 \in p$.

(b) Say that $p \subseteq$ Seq is a perfect tree if:

(i) If $s \in p$, then $s\lceil\alpha \in p$ for every $\alpha$.

(ii) If $\alpha<\kappa$ is a limit ordinal, $s \in{ }^{\alpha} 2$, and $s \uparrow \beta \in p$ for every $\beta<\alpha$, then $s \in p$.

(iii) If $s \in p$, then there is a $t \in p$ with $t \supseteq s$ such that $t$ splits in $p$.

Our definition of perfect trees follows closely [6], with one exception: unlike [6], Definition 1.1(b)(iv), the splitting nodes of $p$ need not be closed.

We consider a notion of forcing $P$ that consists of (some) perfect trees, with the ordering $p \leq q$ iff $p \subseteq q$. Below we formulate several properties of $P$ that guarantee that the proof of minimality for Sacks' forcing generalizes to forcing with $P$.

Definition 2.2. (a) If $p$ is a perfect tree and $s \in p$, set

$$
p_{s}=\{t \in p: s \subseteq t \text { or } t \subseteq s\} ;
$$

$p_{s}$ is a restriction of $p$. A set $P$ of perfect trees is closed under restrictions if for every $p \in P$ and every $s \in p, p_{s} \in P$. If $p_{s}=p$, then $s$ is a stem of $p$.

(b) For each $s \in$ Seq, let $o(s)$ denote the domain (length) of $s$. If $s \in p$ and $o(s)$ is a successor ordinal, $s$ is a successor node of $p$; if $o(s)$ is a limit ordinal, $s$ is a limit node of $p$. If $s$ is a limit node of $p$ and $\{\alpha<o(s): s\lceil\alpha$ splits in $p\}$ is cofinal in $o(s), s$ is a limit of splitting nodes.

(c) Let $p$ be a perfect tree and let $A$ be a nonempty set of mutually incomparable successor nodes of $p$. If, for each $s \in A, q(s)$ is a perfect tree with stem $s$ and $q(s) \leq p_{s}$, let

$$
q=\{t \in p: \text { if } t \supseteq s \text { for some } s \in A, \text { then } t \in q(s)\}
$$

We call the perfect tree $q$ the amalgamation of $\{q(s): s \in A\}$ into $p$. A set $P$ of perfect trees is closed under amalgamations if, for every $p \in P$, every set $A$ of incomparable successor nodes of $p$ and every $\{q(s): s \in A\} \subset P$ with $q(s) \leq p_{s}$, the amalgamation is in $P$.

Definition 2.3. (a) A set $P$ of perfect trees is $\kappa$-closed if for every $\gamma<\kappa$ and every decreasing sequence $\left\langle p_{\alpha}: \alpha<\gamma\right\rangle$ in $P, \bigcap_{\alpha<\gamma} p_{\alpha} \in P$. 
(b) If $\left\langle p_{\alpha}: \alpha<\kappa\right\rangle$ is a decreasing sequence of perfect trees such that

(i) if $\delta$ is a limit ordinal, then $p_{\delta}=\bigcap_{\alpha<\delta} p_{\alpha}$, and

(ii) for every $\alpha, p_{\alpha+1} \cap{ }^{\alpha} 2=p_{\alpha} \cap{ }^{\alpha} 2$,

then $\left\langle p_{\alpha}: \alpha<\kappa\right\rangle$ is called a fusion sequence. A set $P$ is closed under fusion if, for every fusion sequence $\left\langle p_{\alpha}: \alpha<\kappa\right\rangle$ in $P, \bigcap_{\alpha<\kappa} p_{\alpha} \in P$.

The following theorem is a generalization of Sacks' Theorem from [9] to the uncountable case:

Theorem 2.4. Let $P$ be a set of perfect trees and assume that $P$ is closed under restrictions and amalgamations, $\kappa$-closed, and closed under fusion. If $G$ is $P$ generic over $V$, then $G$ is minimal over $V$; namely if $X \in V[G]$ and $X \notin V$, then $G \in V[X]$. Moreover, $V[G]$ has no new bounded subsets of $\kappa$, and $G$ can be coded by a subset of $\kappa$.

Proof. The proof follows as much as in 9 . Given a name $\dot{X}$ for a set of ordinals and a condition $p \in P$ that forces $\dot{X} \notin V$, one finds a condition $q \leq p$ and a set of ordinals $\left\{\gamma_{s}: s\right.$ splits in $\left.q\right\}$ such that $q_{s-0}$ and $q_{s \frown 1}$ both decide $\gamma_{s} \in \dot{X}$, but in opposite ways. The generic branch can then be recovered from the interpretation of $\dot{X}$.

To construct $q$ and $\left\{\gamma_{s}\right\}$ one builds a fusion sequence $\left\{p_{\alpha}: \alpha<\kappa\right\}$ as follows. Given $p_{\alpha}$, let $Z=\left\{s \in p_{\alpha}: o(s)=\alpha\right.$ and $s$ splits in $\left.p_{\alpha}\right\}$. For each $s \in Z$, let $\gamma_{s}$ be an ordinal such that $\left(p_{\alpha}\right)_{s}$ does not decide $\gamma_{s} \in \dot{X}$. Let $q(s \frown 0) \leq\left(p_{\alpha}\right)_{s \frown 0}$ and $q(s \frown 1) \leq\left(p_{\alpha}\right)_{s \frown 1}$ be conditions that decide $\gamma_{s} \in \dot{X}$ in opposite ways. Then let $p_{\alpha+1}$ be the amalgamation of $\{q(s \frown i): s \in Z$ and $i=0,1\}$ into $p_{\alpha}$. Finally, let $q=\bigcap_{\alpha<\kappa} p_{\alpha}$.

In [6] it is postulated that the splitting nodes along any branch of a perfect tree form a closed unbounded set. This guarantees that the set of all such trees is $\kappa$ closed and closed under fusion (Lemmas 1.2 and 1.4 in [6]). It turns out that a less restrictive requirement suffices.

Definition 2.5. Let $S \subset \kappa$ be a stationary set. A perfect tree $p \in P$ is $S$-perfect if whenever $s$ is a limit of splitting nodes of $p$ such that $o(s) \in S$, then $s$ splits in $p$.

Lemma 2.6. (a) If $\left\langle p_{\alpha}: \alpha<\gamma\right\rangle, \gamma<\kappa$, is a decreasing sequence of $S$-perfect trees, then $\bigcap_{\alpha<\gamma} p_{\alpha}$ is a perfect tree.

(b) If $\left\langle p_{\alpha}: \alpha<\kappa\right\rangle$ is a fusion sequence of $S$-perfect trees, then $\bigcap_{\alpha<\kappa} p_{\alpha}$ is a perfect tree.

Proof. (a) Let $p=\bigcap_{\alpha<\gamma} p_{\alpha}$. The only condition in Definition 2.1 (b) that needs to be verified is (iii): for every $s \in p$ find $t \supseteq s$ that splits in $p$. First it is straightforward to find a branch $f \in{ }^{\kappa} 2$ through $p$ such that $s$ is an initial segment of $f$.

Second, it is equally straightforward to see that, for each $\alpha<\gamma$, the set of all $\beta$ such that $f\left\lceil\beta\right.$ splits in $p_{\alpha}$ is unbounded in $\kappa$. Thus for each $\alpha<\gamma$ let $C_{\alpha}$ be the closed unbounded set of all $\delta$ such that $f\left\lceil\delta\right.$ is a limit of splitting nodes in $p_{\alpha}$. Let $\delta \geq o(s)$ be an ordinal in $\bigcap_{\alpha<\gamma} C_{\alpha} \cap S$. Then for each $\alpha<\gamma, t=f\lceil\delta$ is a limit of splitting nodes in $p_{\alpha}$, and hence $t$ splits in $p_{\alpha}$. Therefore $t$ splits in $p$.

(b) Let $p=\bigcap_{\alpha<\kappa} p_{\alpha}$ and again, check (iii). Let $s \in p$, and let $f \in{ }^{\kappa} 2$ be a branch trough $p$. For each $\alpha<\gamma$ let $C_{\alpha}$ be the club of all $\delta$ such that $f \uparrow \delta$ is a limit of splitting nodes in $p_{\alpha}$. Let $\delta \geq o(s)$ be an ordinal in $\Delta_{\alpha<\kappa} C_{\alpha} \cap S$ and let $t=f\lceil\delta$. 
If $\alpha<\delta$, then $t$ splits in $p_{\alpha}$, and therefore $t$ splits in $p_{\delta}$. Since $p_{\delta+1} \cap{ }^{\delta} 2=p_{\delta} \cap{ }^{\delta} 2$, we have $t \in p_{\delta+1}$, and since $p_{\delta+1}$ is $S$-perfect, $t$ splits in $p_{\delta+1}$. If $\alpha>\delta+1$, then $p_{\alpha} \cap{ }^{\delta+1} 2=p_{\delta+1} \cap{ }^{\delta+1} 2$, and so $t$ splits in $p_{\alpha}$. Hence $t$ splits in $p$.

This is trivial, but note that the limit condition $p$ (in both (a) and (b)) is not only perfect but $S$-perfect as well.

\section{The notion of Forcing For Which $B(P)$ Is Rigid}

We now define a set $P$ of perfect $\kappa$-trees that is closed under restrictions and amalgamations, $\kappa$-closed, and closed under fusion, with the additional property that the complete Boolean algebra $B(P)$ is rigid. That completes a proof of the Main Theorem.

Let $S$ and $S_{\xi}, \xi<\kappa$, be mutually disjoint stationary subsets of $\kappa$, such that for all $\xi<\kappa$, if $\delta \in S_{\xi}$, then $\delta>\xi$.

Definition 3.1. The forcing notion $P$ is the set of all $p \subseteq$ Seq such that

(1) $p$ is a perfect tree;

(2) $p$ is $S$-perfect, i.e. if $s$ is a limit of splitting nodes of $p$ and $o(s) \in S$, then $s$ splits in $p$

(3) For every $\xi<\kappa$, if $s$ is a limit of splitting nodes of $p$ with $o(s) \in S_{\xi}$ and if $s(\xi)=0$, then $s$ splits in $p$.

The set $P$ is ordered by $p \leq q$ iff $p \subseteq q$.

Clearly, $P$ is closed under restrictions and amalgamations. By Lemma 2.6, the intersection of either a decreasing short sequence or of a fusion sequence in $P$ is a perfect tree, and since both properties (2) and (3) are preserved under arbitrary intersections, we conclude that $P$ is also $\kappa$-closed and closed under fusion.

We conclude the proof by showing that $B(P)$ is rigid.

Lemma 3.2. If $\pi$ is a nontrivial automorphism of $B(P)$, then there exist conditions $p$ and $q$ with incomparable stems such that $\pi(p)$ and $q$ are compatible (in $B(P))$.

Proof. Let $\pi$ be a nontrivial automorphism. It is easy to find a nonzero element $u \in B$ such that $\pi(u) \cdot u=0$. Let $p_{1} \in P$ be such that $p_{1} \leq u$, and let $q_{1} \in P$ be such that $q_{1} \leq \pi\left(p_{1}\right)$. As $p_{1}$ and $q_{1}$ are incompatible, there exists some $t \in q_{1}$ such that $t \notin p_{1}$. Let $q=\left(q_{1}\right)_{t}$. Then let $p_{2} \in P$ be such that $p_{2} \leq \pi^{-1}(q)$, and again, there exists some $s \in p_{2}$ such that $s \notin q$. Let $p=\left(p_{2}\right)_{s}$. Now $s$ and $t$ are incomparable stems of $p$ and $q$, and $\pi(p) \leq q$.

To prove that $B(P)$ has no nontrivial automorphism, we introduce the following property $\varphi(\xi)$.

Definition 3.3. Let $\xi<\kappa$; we say that $\xi$ has property $\varphi$ if and only if for every function $f: \kappa \rightarrow 2$ there exist a function $F:$ Seq $\rightarrow 2$ in $V$ and a club $C \subset \kappa$ such that, for every $\delta \in C \cap S_{\xi}, f(\delta)=F(f\lceil\delta)$.

Lemma 3.4. Let $t_{0} \in S e q$ and let $\xi=o\left(t_{0}\right)$.

(a) Every condition with stem $t_{0} 0$ forces $\neg \varphi(\xi)$.

(b) Every condition with stem $t_{0}^{\frown} 1$ forces $\varphi(\xi)$. 
Proof. (a) Let $\dot{f}$ be the name for the generic branch $f_{G}: \kappa \rightarrow 2$ (i.e. $f_{G}=$ $\bigcup\{s \in$ Seq $: s \in p$ for all $p \in G\})$; this will be the counterexample for $\varphi(\xi)$. Let $F$ be a function, $F$ : Seq $\rightarrow 2$, let $\dot{C}$ be a name for a club and let $p \in P$ be such that $t_{0}^{-0} 0$ is a stem of $p$. We shall find a $\delta \in S_{\xi}$ and $q \leq p$ such that $q \Vdash(\delta \in \dot{C}$ and $\dot{f}(\delta) \neq F(\dot{f}\lceil\delta))$.

We construct a fusion sequence $\left\langle p_{\alpha}: \alpha<\kappa\right\rangle$, starting with $p$, so that for each $\alpha$, if $s \in p_{\alpha+1}$ and $o(s)=\alpha+1$, then $\left(p_{\alpha+1}\right)_{s}$ decides the value of the $\alpha$ th element of $\dot{C}$; we call this value $\gamma_{s}$. (We obtain $p_{\alpha+1}$ by amalgamation into $p_{\alpha}$.) Let $r=\bigcap_{\alpha<\kappa} p_{\alpha}$.

Let $b$ be a branch through $r$, and let $s_{\alpha}=b\lceil\alpha$ for all $\alpha$. There exists a $\delta \in S$ such that $s_{\delta}$ is a limit of splitting nodes of $r$, and such that, for every $\alpha<\delta$, $\gamma_{s_{\alpha+1}}<\delta$. Since $s_{\delta}(\xi)=0, s_{\delta}$ splits in $r$, and $r_{s_{\delta}} \Vdash \delta \in \dot{C}$.

Now if $F\left(s_{\delta}\right)=i$, it is clear that $g=r_{s_{\delta}(1-i)}$ forces $\dot{f}\left\lceil\delta=s_{\delta}\right.$ and $\dot{f}(\delta)=1-i$.

(b) Let $\dot{f}$ be a name for a function from $\kappa$ to 2 , and let $p$ be a condition with stem $t_{0} 1$ that forces $\dot{f} \notin V(\varphi(\xi)$ holds trivially for those $f$ that are in $V)$. We shall construct a condition $q \leq p$ and collections $\left\{h_{s}: s \in Z\right\}$ and $\left\{i_{s}: s \in Z^{\prime}\right\}$, where $Z$ is the set of all limits of splitting nodes in $q$ and $Z^{\prime}=\left\{s \in Z: o(s) \in S_{\xi}\right\}$, such that

(i) For each $s \in Z, h_{s} \in$ Seq and $o\left(h_{s}\right)=o(s)$; if $o(s)=\alpha$, then $q_{s} \Vdash \dot{f}\left\lceil\alpha=h_{s}\right.$.

(ii) If $s, t \in Z, o(s)=o(t)=\alpha$, and $s \neq t$, then $h_{s} \neq h_{t}$.

(iii) For each $s \in Z^{\prime}, i_{s}=0$ or $i_{s}=1$; if $o(s)=\delta$, then $q_{s} \Vdash \dot{f}(\delta)=i_{s}$.

Then we define $F$ by setting $F\left(h_{s}\right)=i_{s}$, for all $s \in Z^{\prime}$ (and $F(h)$ arbitrary for all other $h \in \mathrm{Seq}$ ); this is possible because of (ii). We claim that $q$ forces that, for some club $C, \dot{f}(\delta)=F\left(\dot{f}\lceil\delta)\right.$ for all $\delta \in C \cap S_{\xi}$. (This will complete the proof.)

To prove the claim, let $G$ be a generic filter with $q \in G$, let $g$ be the generic branch $(g=\bigcup\{s: s \in p$ for all $p \in G\})$, and let $f$ be the $G$-interpretation of $\dot{f}$. Let $C$ be the set of all $\alpha$ such that $g \uparrow \alpha$ is the limit of splitting nodes in $q$. If $\delta \in C \cap S_{\xi}$, let $s=g \uparrow \delta$; then $s \in Z^{\prime}, f \uparrow \delta=h_{s}$ and $f(\delta)=i_{s}$. It follows that $f(\delta)=F(f\lceil\delta)$.

To construct $q, h_{s}$ and $i_{s}$, we build a fusion sequence $\left\langle p_{\alpha}: \alpha<\kappa\right\rangle$ starting with $p_{0}$. We take $p_{\alpha}=\bigcap_{\beta<\alpha} p_{\beta}$ when $\alpha$ is a limit ordinal, and construct $p_{\alpha+1} \leq p_{\alpha}$ such that $p_{\alpha+1} \cap{ }^{\alpha} 2=p_{\alpha} \cap{ }^{\alpha} 2$. For each $\alpha$, we satisfy the following requirements:

(3.6) For all $s \in p_{\alpha}$, if $o(s)<\alpha$, then:

(i) If $s$ is a limit of splitting nodes in $p_{\alpha}$ and $o(s) \in S_{\xi}$, then $s$ does not split in $p_{\alpha}$.

(ii) If $s$ does not split in $p_{\alpha}$, then $\left(p_{\alpha}\right)_{s}$ decides the value of $\dot{f}(o(s))$.

(iii) If $s$ splits in $p_{\alpha}$, let $\gamma_{s}$ be the least $\gamma$ such that $\left(p_{\alpha}\right)_{s}$ does not decide $\dot{f}(\gamma)$. Then $\left(p_{\alpha}\right)_{s \frown 0}$ and $\left(p_{\alpha}\right)_{s \frown 1}$ decide $\dot{f}\left(\gamma_{s}\right)$ in opposite ways, and both $\left(p_{\alpha}\right)_{s \frown 0}$ and $\left(p_{\alpha}\right)_{s \frown 1}$ have stems of length greater than $\gamma_{s}$.

Note that if $p_{\alpha}$ satisfies (iii) for a given $s$, then every $p_{\beta}, \beta>\alpha$, satisfies (iii) for this $s$, with the same $\gamma_{s}$. Also (by induction on $o(s)$ ), we have $\gamma_{s} \geq o(s)$. Clearly, if $\alpha$ is a limit ordinal and each $p_{\beta}, \beta<\alpha$, satisfies (3.6), then $p_{\alpha}$ also satisfies (3.6). We show below how to obtain $p_{\alpha+1}$ when we have already constructed $p_{\alpha}$.

Now let $q=\bigcap_{\alpha<\kappa} p_{\alpha}$, and let us verify that $q$ satisfies (3.5). So let $\alpha$ be a limit ordinal, and let $Z_{\alpha}=\{t \in q: t$ is a limit of splitting nodes in $q$ and $o(t)=\alpha\}$. If 
$t \in Z_{\alpha}$, then $t$ is a limit of splitting nodes of $p_{\alpha}$. It follows from (3.6) (ii) and (iii) that $\left(p_{\alpha}\right)_{t}$ decides $\dot{f}\left\lceil\alpha\right.$, and we let $h_{t}$ be this sequence. If $t_{1} \neq t_{2}$ are in $Z_{\alpha}$, let $s=t_{1} \cap t_{2}$. By (3.6) (iii) we have $\gamma_{s}<\alpha$ (because there exist $s_{1}$ and $s_{2}$ such that $s \subset s_{1} \subset t_{1}, s \subset s_{2} \subset t_{2}$ and both $s_{1}$ and $s_{2}$ split in $\left.p_{\alpha}\right)$. It follows that $h_{t_{1}} \neq h_{t_{2}}$. If $\alpha \in S_{\xi}$ and $s \in Z_{\alpha}$, then by (3.6) (i), $s$ does not split in $p_{\alpha+1}$ and so $\left(p_{\alpha+1}\right)_{s}$ decides $\dot{f}(\alpha)$; we let $i_{s}$ be this value. These $h_{t}$ and $i_{s}$ satisfy (3.5) for the condition $q$.

It remains to show how to obtain $p_{\alpha+1}$ from $p_{\alpha}$. Thus assume that $p_{\alpha}$ satisfies (3.6). First let $r \leq p_{\alpha}$ be the following condition such that $r \cap{ }^{\alpha} 2=p_{\alpha} \cap{ }^{\alpha} 2$ : If $\alpha \notin S_{\xi}$, let $r=p_{\alpha}$; if $\alpha \in S_{\xi}$, consider all $s \in p_{\alpha}$ with $o(s)=\alpha$ that are limits of splitting nodes, and replace each $\left(p_{\alpha}\right)_{s}$ by a stronger condition $r(s)$ such that $s$ does not split in $r(s)$. For all other $s \in p_{\alpha}$ with $o(s)=\alpha$, let $r(s)=\left(p_{\alpha}\right)_{s}$. Let $r$ be the amalgamation of the $r(s)$; the tree $r$ is a condition because $s(\xi)=1$ for all $s \in p_{\alpha}$ with $o(s)=\alpha$.

Now consider all $s \in r$ with $o(s)=\alpha$. If $s$ does not split in $r$, let $t$ be the successor of $s$ and let $q(t) \leq r_{t}$ be some condition that decides $\dot{f}(\alpha)$. If $s$ splits in $r$, let $t_{1}$ and $t_{2}$ be the two successors of $s$, and let $\gamma_{s}$ be the least $\gamma$ such that $\dot{f}(\gamma)$ is not decided by $r_{s}$. Let $q\left(t_{1}\right) \leq r_{t_{1}}$ and $q\left(t_{2}\right) \leq r_{t_{2}}$ be conditions that decide $\dot{f}\left(\gamma_{s}\right)$ in opposite ways, and such that they have stems of length greater than $\gamma_{s}$.

Now we let $p_{\alpha+1}$ be the amalgamation of all the $q(t), q\left(t_{1}\right), q\left(t_{2}\right)$ into $r$. Clearly, $p_{\alpha+1} \cap{ }^{\alpha} 2=r \cap{ }^{\alpha} 2=p_{\alpha} \cap{ }^{\alpha} 2$. The condition $p_{\alpha+1}$ satisfies (3.6) (i) because $p_{\alpha} \leq r$. It satisfies (ii) because if $s$ does not split and $o(s)=\alpha$, then $\left(p_{\alpha+1}\right)_{s}=q(t)$ where $t$ is the successor of $s$. Finally, it satisfies (iii), because if $s$ splits and $o(s)=\alpha$, then $\left(p_{\alpha+1}\right)_{s \frown 0}=q\left(t_{1}\right)$ and $\left(p_{\alpha+1}\right)_{s \frown 1}=q\left(t_{2}\right)$ where $t_{1}$ and $t_{2}$ are the two successors of $s$.

We now complete the proof that $B(P)$ is rigid.

Theorem 3.7. The complete Boolean algebra $B(P)$ has no nontrivial automorphism.

Proof. Assume that $\pi$ is a nontrivial automorphism of $B(P)$. By Lemma 3.2 there exist conditions $p$ and $q$ with incomparable stems $s$ and $t$ such that $\pi(p)$ and $q$ are compatible. Let $t_{0}=s \cap t$ and let $\xi=o\left(t_{0}\right)$. Hence $t_{0}^{-0}$ and $t_{0}^{-1}$ are stems of the two conditions and by Lemma 3.4, one forces $\varphi(\xi)$ and the other forces $\neg \varphi(\xi)$. This is a contradiction because $\pi(p)$ forces the same sentences that $p$ does, and $\pi(p)$ is compatible with $q$.

\section{REFERENCES}

1. M. Bekkali and R. Bonnet, Rigid Boolean Algebras, in: "Handbook of Boolean Algebras" vol. 2 (J. D. Monk and R. Bonnet, eds.,) p. 637-678, Elsevier Sci. Publ. 1989. CMP 21:10

2. T. Jech, A propos d'algèbres de Boole rigide et minimal, C. R. Acad. Sc. Paris, série A, 274 (1972), 371-372. MR 44:6569

3. T. Jech, Simple complete Boolean algebras, Israel J. Math. 18 (1974), 1-10. MR 50:4300

4. T. Jech and S. Shelah, A complete Boolean algebra that has no proper atomless complete subalgebra, J. of Algebra 182 (1996), 748-755. MR 97j:03109

5. R. B. Jensen, Definable sets of minimal degree, in: Mathematical logic and foundations of set theory. (Y. Bar-Hillel, ed.) p. 122-128, North-Holland Publ. Co. 1970. MR 46:5130

6. A. Kanamori, Perfect set forcing for uncountable cardinals, Annals Math. Logic 19 (1980), 97-114. MR 82i:03061

7. K. McAloon, Consistency results about ordinal definability, Annals Math. Logic 2 (1970), 449-467. MR 45:1753 
8. K. McAloon, Les algèbres de Boole rigides et minimales, C. R. Acad. Sc. Paris, série A 272 (1971), 89-91. MR 42:7491]

9. G. Sacks, Forcing with perfect closed sets, in: "Axiomatic set theory," (D. Scott, ed.) Proc. Symp. Pure Math. 13 (1), pp. 331-355, AMS 1971. MR 43:1827]

10. S. Shelah, Why there are many nonisomorphic models for unsuperstable theories, in: Proc. Inter. Congr. Math., Vancouver, vol. 1, (1974) pp. 259-263. MR 54:10008

Department of Mathematics, The Pennsylvania State University, 218 McAllister Bldg., University Park, Pennsylvania 16802

E-mail address: jech@math.psu.edu

Current address: Center for Theoretical Study, Jilská 1, 11000 Praha 1, Czech Republic

E-mail address: jech@cts.cuni.cz

Institute of Mathematics, The Hebrew University of Jerusalem, 91904 Jerusalem, ISRAEL

E-mail address: shelah@math.rutgers.edu 